\title{
The Significance of Particle's Density in Quantum Field Theory
}

\author{
Eliahu Comay \\ Charactell Ltd., Tel-Aviv, Israel \\ Email: elicomay@post.tau.ac.il
}

How to cite this paper: Comay, E. (2021) The Significance of Particle's Density in Quantum Field Theory. Open Access Library Journal, 8: e7384.

https://doi.org/10.4236/oalib.1107384

Received: April 3, 2021

Accepted: May 23, 2021

Published: May 26, 2021

Copyright $\odot 2021$ by author(s) and Open Access Library Inc.

This work is licensed under the Creative Commons Attribution International License (CC BY 4.0).

http://creativecommons.org/licenses/by/4.0/

\section{(c) (i) Open Access}

\begin{abstract}
The paper emphasizes the significance of density of an elementary massive quantum particle. In quantum field theory, a quantum function of an elementary particle takes the form of $\psi(\boldsymbol{r}, t)$. This kind of function is used for putting the inner product of the corresponding Hilbert space in the form of an appropriate integral, and the inner product of a function with itself depends on the particle's density. Density also affects the multi-particle Fock space, because this space relies on single-particle Hilbert space. This work shows a new reason where a coherent theoretical expression for the density of an elementary particle is required: A theoretical description of experiments that measure the transition of unstable states and the decay of an elementary quantum particle. This new aspect of density strengthens its meaning in quantum theories. The usefulness of this outcome is shown in its application to the decay of the muon and the electroweak's $W^{ \pm}, Z$ particles. It turns out that the Dirac theory provides a consistent description of the muon decay. In contrast, the electroweak theory fails to explain the decay of the $W^{ \pm}, Z$ particles.
\end{abstract}

\section{Subject Areas}

Particle Physics

\section{Keywords}

Quantum Theories, The Hilbert and the Fock Spaces, Density of an Elementary Quantum Particle, Unstable Particles, Decay of the Muon and the $W^{ \pm}, Z$

\section{Introduction}

This work analyzes the significance of the Fock space in Quantum Field Theories (QFT). An observation of contemporary textbooks indicates the timely need for 
an examination of this issue. There are several QFT textbooks that discuss this concept as an element of the mathematical structure of the theory (see e.g. [1] [2] [3]). On the other hand, there are too many QFT textbooks that refrain from this discussion. This work shows the significance of the Fock space as an element of a coherent description of processes that belong to the QFT domain of validity.

The significance of particle density is another issue that is analyzed in this work. It is already known that charge density is a fundamental element of Maxwellian electrodynamics (see [4], p. 75). Evidently, a quantum expression for the charge density of an elementary particle is proportional to the particle's density. Hence, particle density is an inherent element of QED.

Measurements of the decay of some elementary massive particles are analyzed below. The quantum description of a decaying particle uses destruction and creation operators, and these operators change the occupation number of every relevant particle in the Fock space. It is explained below how density affects the Hilbert space that is an element of the Fock space.

A new aspect of particle density is pointed out hereinafter, and it emphasizes the role of this issue in quantum theories. This work analyzes the need for particle density of a quantum description of the time-evolution of unstable states. This novel requirement is applied in an analysis of quantum transitions of states that pertain to the Dirac theory of an elementary spin-1/2 massive particle. The same procedure is applied to the electroweak theory of the $W^{ \pm}, Z$ particles.

The main objective of this work is to upgrade the meaning of density of an elementary quantum particle. At present some textbooks do not discuss this topic at all, whereas others do not emphasize its crucial role in the theory's structure. It is proved below that a coherent expression for the density of an elementary quantum particle is a necessary condition for every QFT.

Units where $\hbar=c=1$ are used. Greek indices run from 0 to 3. Most formulas take the standard form, and relativistic covariant expressions are used. The metric is diagonal and its entries are $(1,-1,-1,-1)$. The second section presents some principles that are utilized in this work. The third and the fourth sections briefly describe the Hilbert and the Fock spaces, respectively. The fifth section analyzes quantum transitions of states of Dirac particles. The sixth section points out discrepancies in the electroweak description of the decay of the $W^{ \pm}, Z$ particles. The last section summarizes this work.

\section{Principles Used in This Article}

Physics is a mature science whose theories are written in mathematical language. During the development of theoretical physics, quite a few general principles are now recognized as solid elements of any physical theory. Although it is a good idea to test and retest every principle of this kind, this work does not aim to do that.

Below, some principles are briefly described, and the main objective of doing that is to help readers see the consistency of the analysis. Few theoretical ele- 
ments that are derived from these principles are also shown.

PR.1 The Generalized Correspondence Principle (GCP).

This work is not the right place for a comprehensive discussion of this important topic (see pp. 1-6 of [5]). Hence, only topics that are required for a later discussion are pointed out here.

(a) Textbooks on quantum mechanics (QM) explain the correspondence between QM and classical physics: "classical mechanics must therefore be a limiting case of quantum mechanics" (see [6], p. 84). A further discussion can be found in [7], (see pp. 25-27, 137, 138) as well as in many other QM textbooks.

(b) Another well-known textbook states the correspondence between QFT and QM: "First, some good news: quantum field theory is based on the same quantum mechanics that was invented by Schroedinger, Heisenberg, Pauli, Born, and others in 1925-1926, and has been used ever since in atomic, molecular, nuclear and condensed matter physics" (see [8], p. 49).

These arguments mean that there is correspondence between the three physical theories:

$$
\mathrm{QFT} \rightarrow \mathrm{QM} \rightarrow \text { Classical physics }
$$

An important point of the correspondence relationships is that the appropriate limit of the value of a variable of the higher rank theory should agree with the corresponding value found in circumstances where the lower rank theory is $\mathrm{OK}$. It means that:

Conclusion \#1: QFT must have a coherent expression for every variable that is defined and measured by QM and by classical physics as well.

\section{PR.2 The Variational Principle (VP).}

VP is now regarded as the cornerstone of the QFT of every specific elementary particle. Consider the VP and a Lagrangian density of the form

$$
\mathcal{L}\left(\psi(x), \psi(x)_{, \mu}\right)
$$

where $x$ denotes the four space-time coordinates, and $\psi(x) \equiv \psi(\boldsymbol{r}, t)$. There is now a general agreement that: "All field theories used in current theories of elementary particles have Lagrangians of this form" (see [8], p. 300). This principle is adopted in this work.

Several consequences of the VP are used hereinafter. As stated above, an important element of this work is a discussion of the quantum expression for particle density. In a relativistic language, a particle's density is the 0 -component of its 4-current (see [4], p. 75). The Noether theorem depends on the VP. One of its results is a general expression for the particle's conserved 4-current

$$
j^{\mu}=\frac{\partial \mathcal{L}}{\partial \psi_{, \mu}} \psi
$$

(see [9], p. 314). 
The 4-current of a Dirac particle is required for a later discussion. The QED Lagrangian density (see e.g. [10], p. 84, [11], p. 78)

$$
\mathcal{L}_{Q E D}=\bar{\psi}\left[\gamma^{\mu}\left(i \partial_{\mu}-e A_{\mu}\right)-m\right] \psi-\frac{1}{16 \pi} F_{\mu \nu} F^{\mu \nu} .
$$

is used for this end. The general expression of the Noether theorem for the 4-current of a quantum particle (3) and the QED Lagrangian density (4) yield the Dirac particle's 4-current

$$
j^{\mu}=\bar{\psi} \gamma^{\mu} \psi
$$

(see, e.g. [12], pp. 23, 24). The 0-component of (5) is the density of a Dirac particle

$$
\rho=\psi^{\dagger} \psi
$$

This relation yields a coherent quantum expression for the position of a Dirac particle whose state is $\psi_{i}(\boldsymbol{r}, t)$

$$
\langle\boldsymbol{r}(t)\rangle=\int \psi_{i}^{\dagger}(\boldsymbol{r}, t) \boldsymbol{r} \psi_{i}(\boldsymbol{r}, t) \mathrm{d}^{3} r
$$

(see e.g. [7], p. 24). These properties of the Dirac theory are used below.

\section{The Hilbert Space}

For the simplification of the notation and a later application, the single-particle quantum functions used herein take the form $\psi(\boldsymbol{r}, t)$, briefly denoted $\psi(x)$, where $x$ is the four space-time coordinates. This form is used by the VP of QFT (see item 2). Textbooks show how these functions can be used for a definition of a Hilbert space (see e.g. [8], pp. 49, 50; [13], pp. 164-166). The main points of a Hilbert space and its applicability to quantum theories are very briefly described herein.

A set $H$ of complex functions $\psi_{i}$ may compose a Hilbert space if the following properties hold:

P.1 The integrability requirement says that for every $\psi_{i}$ the integral

$$
Q=\int \psi_{i}^{\dagger} \psi_{i} \mathrm{~d}^{3} r
$$

converges, and $Q$ is a real non-negative number.

P.2 $Q=0$ if and only if $\psi_{i} \equiv 0$.

P.3 The linearity requirement says that if $\psi_{i}, \psi_{j}$ belong to $H$ and $a, b$ are complex numbers then

$$
\psi=a \psi_{i}+b \psi_{j}
$$

belongs to $H$.

P.4 A scalar product is defined for every pair of functions of $H$

$$
\left\langle\psi_{j} \mid \psi_{i}\right\rangle \equiv \int \psi_{j}^{\dagger} \psi_{i} \mathrm{~d}^{3} r
$$

Variables of classical physics take a mathematically real value. Moreover, mathematically real eigenvalues are an important property of a Hermitian operator. Hence, in order to satisfy the classical limit, a quantum variable that corresponds 
to a classical quantity is an eigenvalue of a Hermitian operator $\hat{O}_{k}$ that operates on functions of $H$. If $\psi_{i}$ is an eigenfunction of $\hat{O}_{k}$ then the corresponding eigenvalue $q_{k}$ of $\psi_{i}$ is

$$
q_{k}=\int \psi_{i}^{\dagger} \hat{O}_{k} \psi_{i} \mathrm{~d}^{3} r
$$

\section{The Fock Space}

Many physical systems comprise more than one particle. The Fock space is constructed for a theoretical description of these systems. The Fock space is an appropriate sum of Hilbert spaces, where each Hilbert space comprises single-particle quantum functions. As such, it can be used for a description of many-particle states. If the quantum system comprises more than one particle of the same kind $P_{i}$ then the Fock space is antisymmetric with respect to the functions of $P_{i}$ if these particles are fermions and symmetric if they are bosons. In the examples that are discussed in this work there is no more than one particle of any specific type. Therefore, the antisymmetric/symmetric issue is not mentioned hereinafter.

The Fock space is relevant to the decay processes that are discussed in this work.

\section{Transitions of Dirac Particles}

Let us examine two kinds of transitions of Dirac particles.

\subsection{Transition of Electronic States}

Consider the $l=0$ hydrogen atom ground state, called $1 \mathrm{~s}$, and its excited $l=1$ state, called $2 p$. Let $\psi_{s}, \psi_{p}$ respectively denote these states. $\psi_{p}$ is unstable, and its transition is

$$
\psi_{p} \rightarrow \psi_{s} \Phi
$$

where $\Phi$ denotes the function of the outgoing photon. This is an example of a transition from a quantum unstable state to a final stable state, and the process conserves the Dirac particle that belongs to the system. Here are the theoretical elements that explain the transition (12).

Rad.1 The electron has a coherent expression for its 4-current (5), and density is the $j^{0}$ component of a 4-current. Therefore, the electron's density is

$$
\rho_{e}=\psi^{\dagger} \psi
$$

This expression for the electron's density holds for stable and unstable states of the hydrogen atom. It is used for a definition of the inner product of the electron's Hilbert space that comprises the quantum functions of the hydrogen atom (see [13], pp. 164-166).

Rad.2 The Hilbert space enables a coherent expression for operators that apply to the electron's quantum functions (see [8], p. 50). The electron's Hamiltonian is an operator of this kind. 
Rad.3 The $H_{k m}$ entry of the Hamiltonian's matrix element is a crucial quantity in a description of the time-dependent transition between the $k . m$ states (see [7], pp. 195-199). This result explains the process (12).

Conclusion \#2: The electronic state $\psi_{p}$ is unstable and after a cer-
tain period it completes its transition to the state $\psi_{s}$ and a photon.
The electronic functions $\psi_{s}$, and $\psi_{p}$ have a mathematically coherent
expression for density. This expression is used for the inner product of
the electron's Hilbert space, and textbooks show how the required ma-
trix elements of the Hamiltonian can be calculated. This Hamiltonian
describes the transition of $(12)$.

\subsection{The Muon Decay}

Figure 1 illustrates the muon decay. This is a weak-interaction process where one Dirac particle is destroyed and three Dirac particles emerge. Let us examine elements of the experimental measurement of this process.

In principle, each of the outgoing particles is recorded by a specific detector. This detector records the energy-momentum and the detector's space-time point of the arriving particle. As usual, these data include an estimate of the measurement error. This information is used by experimenters to find whether or not two requirements hold: The outgoing particles should emerge from a very small space-time region. Furthermore, experimenters calculate the total invariant energy of the outgoing particles and compare it with the muon's mass. If the results of these tests are satisfactory then the event is recorded as a muon decay.

A theoretical description of this effect must yield coherent expressions for the measured quantities - the energy-momentum and the space-time position of the decaying particle of Figure 1. The muon is a Dirac particle and the Dirac theory provides the required expressions for its density and its energy-momentum. As a quantum theory, it abides by the Heisenberg uncertainty principle. Thus, we have here a Dirac particle that moves in the vacuum. The four components of the spinor of a solution of a free spin-up Dirac particle are (see [12], p. 30)

$$
\psi=\left(1,0, p_{z} / Q, p_{+} / Q\right) S \exp (i(\boldsymbol{k} \cdot \boldsymbol{x}-\omega t)),
$$

where

$$
p_{+}=p_{x}+i p_{y}, Q=E+m, S=\sqrt{Q / 2 m},
$$

and $m, E$ denote respectively the particle's mass and energy. As stated above,

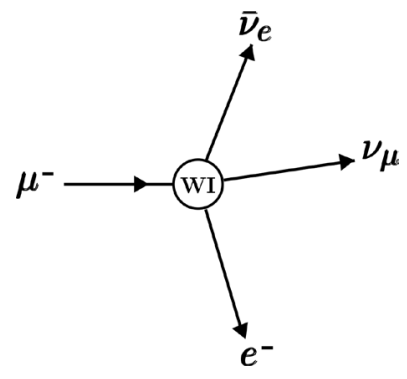

Figure 1 . The $\mu^{-}$decay (see text). 
the density of a quantum particle is the 0-component of its 4-current. The required expression for the Dirac particle's 4-current is shown above (5). The explicit expression for the space-time position of a Dirac particle is (7).

The foregoing points show how the quantum theory of a Dirac particle provides a coherent interpretation of the experiment. This issue is summarized as follows:

1) The Dirac equation is derived from the QED Lagrangian density.

2) The Dirac equation is solved for a free particle and the solution $\psi$ of (14) describes the particle's properties.

3) The Noether theorem is applied to the Dirac Lagrangian density and yields the density expression (5).

4) Section 3 explains how density is used for the construction of a Hilbert space of every Dirac particle and the operators that operate on the quantum functions of this space.

5) Section 4 explains how the Hilbert spaces of all particles of Figure 1 are utilized for the construction of the Fock space of these particles.

6) Weak interaction operators of the Fock space explain the muon decay process.

7) Operators of density and those of the energy-momentum apply to the quantum function $\psi_{k}$ of the decaying particle. This is the theoretical explanation of the elements of the muon decay experiment of Figure 1.

8) In particular, experimenters record the space-time position of the point where an outgoing particle hits its measuring device and the energy-momentum of this particle. This information enables them to determine a quite small space-time region from which all the outgoing particles emerged. Hence, a theory of a decaying particle must provide expressions for its space-time position and its energy-momentum. Relations (7) and (14) respectively satisfy these tasks.

9) Density appears in two different places of the theoretical description of the decay process. It explains the position of the decaying process as well as the position of the outgoing particles at the detectors. It is also used in the inner product of the particles' Hilbert spaces. The Fock space uses these Hilbert spaces and the particle's creation/destruction operators operate on entries of the Fock space.

10) It is interesting to note that the aforementioned arguments are independent of the specific QFT that describes the decaying process.

Conclusion \#3: The Dirac theory of an elementary spin$1 / 2$ particle explains the experimental attributes of a decay of such a particle. The muon decay is an example of this process.

The muon decay of Figure 1 shows an example of the merits of the GCP of (1). The incoming particle moves in the vacuum, and its motion is described by classical physics. In particular, a solution of the classical equations of motion determines the particle's space-time position $\boldsymbol{r}(t)$ and its energy-momentum. 
Conservation of energy-momentum is a well-known effect, and it is utilized in a measurement of the muon decay. The muon decay is explained by the destruction and creation operators of QFT. These operators operate on the Fock space, which is based on the particles' Hilbert spaces of QM. The outgoing particles move in the vacuum and classical physics explains their motion. QFT mediate between the initial and the final states of the muon decay. In order to explain this decay, it must provide coherent expressions for the density of the particles of Figure 1 and their energy-momentum. This is what the GCP of (1) requires.

Conclusion \#4: A QFT of a decaying particle must
provide an expression for its density and its energy-
momentum.

\section{Problems with the Electroweak $W^{ \pm}, Z$ Particles}

The previous section explains why a coherent expression for density is a vital element of a theory of a decaying particle: Experimenters determine a quite small space-time region from which the outgoing particles emerge, and the theory must show that the decaying particle was inside that region.

Let us examine the particles $W^{-}, Z$ which are crucial elements of the electroweak theory [11] [15]. One decay channel of each of these particles is shown on Figure 2. These decays are analogous to the muon decay of Figure 1. Unfortunately, unlike the Dirac theory of elementary spin-1/2 particles, like the muon, the electroweak theory, which is more than 50 years old, still has no coherent expression for the density of each of these particles. A review of the literature proves that indeed, unlike textbooks on the Dirac theory that show an expression for density (see e.g. [8] p. 10; [12] p. 9), electroweak textbooks do not show an analogous expression for the $W^{ \pm}, Z$ particles.

The following lines support this conclusion. The electron's electromagnetic interaction term of the QED Lagrangian density (4) uses the Dirac coherent expression for the charged particle's 4-current. In contrast to the electron, the literature does not show a coherent expression for the electromagnetic interaction of the $W^{ \pm}$. Here is evidence that substantiates this claim. About 20 years after the presentation of the electroweak theory, a group of researchers has published an effective Lagrangian for the electromagnetic interaction of the $W^{ \pm}$(see the beginning of section 2 of [16]). Their effective Lagrangian is also presented in a second article [17]. As a matter of fact, this effective Lagrangian is still used by thousands of people who work in very large research centers like Fermilab and CERN [18] [19].

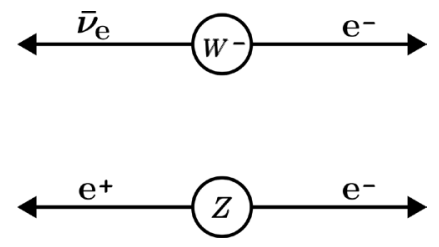

Figure 2. Decay channels of the $W$ and the $Z[14]$. 
It is interesting to compare the theoretical status of a charged Dirac particle with that of the electroweak $W^{ \pm}$. The first article of the Dirac theory of the electron shows a theoretically coherent expression for its electromagnetic interaction [20]. In contrast, many decades after the publication of the electroweak theory, major research centers still use effective expressions for the electromagnetic interaction of the $W^{ \pm}$particles. This unfortunate electroweak situation proves that this theory still lacks a theoretically vital expression for the $W^{ \pm}$electromagnetic interaction and its charge density. As stated in the introduction section, the charge density of an elementary particle is proportional to the particle's density.

Conclusion \#5: The lack of a coherent expression for density proves that the electroweak theory cannot explain the decay of the $W^{ \pm}$and the $Z$ particles.

Conclusion \#6: The electroweak theory is unacceptable.

\section{General Considerations}

QFT textbooks show how the energy-momentum of a quantum particle is derived from its energy-momentum tensor $T^{\mu \nu}$. The entries $T^{0 v}$ represent energy-momentum density, and the spatial integral yields the energy-momentum

$$
P^{v}=\int \mathrm{d}^{3} x T^{0 v}
$$

(see e.g. [8] p. 310; [11], pp. 19, 310). The present section shows how this work provides a self-evident extension to this attribute.

Special relativity combines time and space into one 4 -vector

$$
x^{\mu}=(t, \boldsymbol{r}) .
$$

The same is true for the energy-momentum

$$
p^{\mu}=(E, \boldsymbol{p}) .
$$

Furthermore, coordinates and momenta have very close relationships. For example, the classical canonical equations

$$
\dot{q}_{i}=\partial H / \partial p_{i} ; \dot{p}_{i}=-\partial H / \partial q_{i}
$$

(see [21], p. 337) show symmetrical relationships between these variables. Classical physics terminology describes the close relationships between $x, p_{x}$ by the term conjugate variables (see [21], p. 337). This terminology is also used in quantum mechanics (see [13], p. 32).

The Heisenberg uncertainty relations, which are fundamental elements of quantum theories, relate these variables

$$
\Delta x \cdot \Delta p_{x} \geq \hbar ; \Delta E \cdot \Delta t \geq \hbar
$$

(see [7], p. 7). These expressions indicate another aspect of the close relationships between time-space and energy-momentum.

The present work examines a fundamental QFT process: a decay of an unstable quantum particle, like the muon and the $W^{ \pm}, Z$ particles. Let us point out the elements of a successful measurement of this process. 
1) For every outgoing particle, a detector measures the space-time position where the particle hits the detector and its energy-momentum.

2) The previous data enable a calculation that goes back in time and finds a common space-time region of the outgoing particles. A necessary condition for a positive detection is that the common space-time region is quite small.

3) The same data enable a calculation of the sum of the energy-momentum of the outgoing particles at the region that is determined above. A necessary condition for a positive detection is that the difference between the mass of the decaying particle and the invariant energy of the outgoing particles is quite small.

This work proves that a theoretical interpretation of the decay of a quantum particle should provide adequate expressions for the particle's energy-momentum and its space-time point $\boldsymbol{r}(t)$. Due to the uncertainty relations, density is used for the determination of the position of a quantum particle (7). The requirement for a coherent expression for density is a result of this work. It is analogous to the corresponding requirement of the energy-momentum tensor of (16).

Conclusion \#7: Energy-momentum and time-space are conjugate
variables. This work shows another analogy between these variables:
A QFT of a particle should provide coherent expressions for energy-
momentum density and particle density as well. Spatial integrals of
these densities yield the particle's values of the conjugate variables:
energy-momentum $P^{\mu}$ and space-time $\boldsymbol{r}(t)$.
Conclusion \#8: The outcome of this work is coherently embedded
in general physical principles.

\section{Summary}

This work explains the crucial role of a coherent expression for the density of an elementary massive quantum particle. Particle density is used for the construction of the Hilbert space of QM. A fundamental element of QM is the utilization of operators that apply to the quantum functions that compose this space. The Hilbert space is also used for the construction of the Fock space, where the creation and destruction operators of QFT are used. The present work points out a new relevant argument: The experimental analysis of a decaying particle, like the muon, defines a quite small space-time region from which the outgoing particles emerge. A coherent expression for density enables a theory to show that the decaying particle was inside a given space-time region. This requirement is analogous to that where the invariant mass of the outgoing particles should be equal to the self-mass of the decaying particle. Therefore, a QFT of a decaying elementary quantum particle should provide a coherent expression for its density and its energy-momentum. This outcome makes sense because space-time and energy-momentum are conjugate variables.

Density is the 0 -component of the 4 -current $j^{\mu}$. It is well-known that the Dirac theory of an elementary spin-1/2 massive particle provides a coherent expression for the 4-current (5). In contrast, the electroweak theory has no expression for the density of its $W^{ \pm}, Z$ particles. This discrepancy casts serious doubts 
on the consistency of the electroweak theory.

An examination of the literature indicates the lack of an adequate discussion of the significance of the quantum particle's density. This work will hopefully make a contribution for closing this gap. The same words apply to the Fock space.

\section{Conflicts of Interest}

The author declares no conflicts of interest regarding the publication of this paper.

\section{References}

[1] Schweber, S.S. (1964) An Introduction to Relativistic Quantum Field Theory. Harper \& Row, New York.

[2] Itzykson, C. and Zuber, J.B. (1980) Quantum Field Theory. McGraw-Hill, New York.

[3] Sterman, G. (1993) An Introduction to Quantum Field Theory. Cambridge University Press, Cambridge, 518. https://doi.org/10.1017/CBO9780511622618

[4] Landau, L.D. and Lifshitz, E.M. (2005) The Classical Theory of Fields. Elsevier, Amsterdam.

[5] Rohrlich, F. (2007) Classical Charged Particles. 3rd Edition, World Scientific, New Jersey. https://doi.org/10.1142/6220

[6] Dirac, P.A.M. (1958) The Principles of Quantum Mechanics. Oxford University Press, London.

[7] Schiff, L.I. (1955) Quantum Mechanics. McGraw-Hill, New York.

[8] Weinberg, S. (1995) The Quantum Theory of Fields. Vol. I, Cambridge University Press, Cambridge.

[9] Halzen, F. and Martin, A.D. (1984) Quarks and Leptons, An Introductory Course in Modern Particle Physics. John Wiley, New York.

[10] Bjorken, J.D. and Drell, S.D. (1965) Relativistic Quantum Fields. McGraw-Hill, New York.

[11] Peskin, M.E. and Schroeder, D.V. (1995) An Introduction to Quantum Field Theory. Addison-Wesley, Reading Mass.

[12] Bjorken, J.D. and Drell, S.D. (1964) Relativistic Quantum Mechanics. McGraw-Hill, New York.

[13] Messiah, A. (1967) Quantum Mechanics. Vol. 1, North Holland, Amsterdam.

[14] Particle Data Group (2020) Review of Particle Physics. Progress of Theoretical and Experimental Physics, 2020, Article ID: 083C01.

https://doi.org/10.1093/ptep/ptaa104

[15] Weinberg, S. (1996) The Quantum Theory of Fields. Vol. II, Cambridge University Press, Cambridge.

[16] Hagiwara, K., Peccei, R.D., Zeppenfeld, D. and Hikaso, K. (1987) Probing the Weak Boson Sector in $\mathrm{e}^{+} \mathrm{e}^{-} \rightarrow \mathrm{W}^{+} \mathrm{W}^{-}$. Nuclear Physics B, 282, 253-307.

[17] Hagiwara, K., Woodside, J. and Zeppenfeld, D. (1990) Measuring the WWZ Coupling at the Fermilab Tevatron. Physical Review D, 41, 2113-2119.

[18] D0 Collaboration (2012) Limits on Anomalous Trilinear Gauge Boson Couplings from WW, WZ and $\mathrm{W} \gamma$ Production in $\mathrm{pp}^{-}$Collisions at $\mathrm{s}=1.96 \mathrm{TeV}$. Physics Let- 
ters $B, 718,451-459$. https://doi.org/10.1016/j.physletb.2012.10.062

[19] ATLAS Collaboration (2012) Measurement of the WW Cross Section in sqrt(s) $=7$ $\mathrm{TeV} p p$ Collisions with the ATLAS Detector and Limits on Anomalous Gauge Couplings. Physics Letters B, 712, 289-308. https://doi.org/10.1016/j.physletb.2012.05.003

[20] Dirac, P.A.M. (1928) The Quantum Theory of the Electron. Proceedings of the Royal Society A, 117, 610-324. https://doi.org/10.1098/rspa.1928.0023

[21] Goldstein, H., Poole, C. and Safko, J. (2002) Classical Mechanics. 3rd Edition, Addison Wesley, San Francisco. 